\title{
Traditional and Kalman Filter-Based GNSS Receiver Structures for Ionospheric Scintillation Monitoring and Mitigation
}

\author{
Rafael A. M. Lopes, Felix Antreich, Senior Member, IEEE, and Hélio K. Kuga
}

\begin{abstract}
We assess the estimation of ionospheric scintillation induced effects on global navigation satellite systems (GNSS) receivers for scintillation monitoring and mitigation. We propose a monitoring algorithm composed of frequency locked loops (FLL) observables filtering based on the complementary frequency content of their linear approximation. The algorithm is employed to traditional and Kalman FLLs exploiting their architectures to provide scintillation phase estimates. We also propose a discriminator-based and an extended Kalman phase locked loop (PLL) using independent kinematic process models for the scintillation phase and amplitude to improve robustness to scintillation effects in carrier synchronization. Simulations with synthetic severe scintillation data show the capability of the proposed monitoring algorithm to provide scintillation phase estimates with performance comparable to the proposed Kalman PLLs that explicitly model scintillation effects. Furthermore, we show that the proposed Kalman PLLs to mitigate the scintillation effects achieve advanced Doppler phase and scintillation amplitude estimation performance compared to an extended Kalman PLL with an autoregressive (AR) scintillation process model, with comparable scintillation phase estimation performance. The proposed Kalman PLLs have the additional benefits of not requiring parameter identification of $A R$ models and allowing to constrain the extended filter to handle polarity inversions in scintillation amplitude estimates.
\end{abstract}

Index Terms-Ionospheric scintillation, GNSS, amplitude and phase estimation, frequency locked loop (FLL), Kalman FLL, Kalman PLL.

\section{INTRODUCTION}

$\mathbf{T}$ RANSIENT fluctuations of electron content inside regions of the ionosphere interact with propagating radio waves, resulting in scintillation. For global navigation satellite systems (GNSS) in particular, ionospheric scintillation can have a significant impact on the availability, accuracy, continuity, and integrity of the positioning for users of such systems. The signal processing channels in a GNSS receiver perform carrier and code delay tracking of GNSS satellite signals. Regarding the received signal carrier, ionospheric scintillation is one source of amplitude and phase variations adding up to the line-of-sight (LOS) dynamics, introducing disturbances to GNSS tracking algorithms in the receiver that in many cases cause a reduction of precision in the positioning and eventually in the loss of lock of GNSS signals. Such disturbances may be induced for short periods by severe scintillation,

R. A. M. Lopes and H. K. Kuga are with Departamento de Mecânica e Controle, INPE, São José dos Campos, SP, Brazil e-mail (rafael.lopes@inpe.br and helio.kuga@inpe.br).

Felix Antreich is with Departamento de Telecomunicações, ITA, São José dos Campos, SP, Brazil e-mail (antreich@ieee.org). in equatorial and high-latitude regions [1]. The equatorial region experiences the most significant activity, with severe scintillation resulting in rapid amplitude and phase variations with deep amplitude fades for short periods, depending on factors such as solar activity, geomagnetic activity, latitude, carrier frequency, and daytime [2]. Thereby the detection, monitoring, and mitigation of ionospheric scintillation effects in a GNSS receiver are challenging from the signal processing perspective [1].

High accuracy carrier tracking is vital for positioning accuracy improvement in modern receivers applying carrier-based positioning such as real-time kinematics (RTK) and precise point positioning (PPP) [3], [4]. Especially in the carrier tracking, the scintillation effects are more pronounced and the estimation of the amplitude and phase variations induced by scintillation is important, on the one hand, to increase the tracking loop robustness to those effects, and on the other hand for scintillation detection and monitoring. Originally, the traditional tracking loop structures implemented with phase locked loops (PLL) and/or frequency locked loops (FLL) are not directly providing estimates of scintillation amplitude or phase variations. Their parameters are fixed and adjusted for LOS-only tracking. Robustness can be improved via the tuning of parameters such as noise bandwidth [5] in PLLs or employing an FLL-assisted PLL structure [6], for example. In the case of ionospheric scintillation monitoring, usually, a static receiver with a known position and precise reference oscillator provides estimates of the LOS dynamics with additional accurate information, such as the clock biases and ephemeris of the tracked satellites [7], [8]. It is important to have highly reliable receivers to isolate the scintillation induced effects from the LOS dynamics tracked by the receivers. Scintillation monitoring stations are deployed for different purposes, such as space weather research [9] and as infrastructure for aircraft instrument landing systems [10].

The Kalman filter has been successfully employed in GNSS carrier tracking loops with this intent. Early applications involved suboptimal computation of the Kalman gains by solving only the steady-state Riccati equation, as in [11]. Typically a constant gain Kalman filter structure is chosen when the simplicity of the suboptimal solution is more convenient than the computational load to perform the real-time update of the gains. A Kalman filter PLL was employed with LOS and clock error state variables, and measurement noise adjusted as a function of the carrier-to-noise ratio $\mathrm{C} / \mathrm{N}_{0}$, allowing better phase tracking during the power fades and 
phase dynamics induced by scintillation when compared to a constant bandwidth loop [12]. The introduction of models representing the scintillation dynamics in the received signal into the Kalman filter formulation enabled decoupling both scintillation and LOS contributions to overcome the limitations of techniques that only adjust the level of uncertainty in models accounting for states related to the LOS dynamics, e.g., based on an estimate of $\mathrm{C} / \mathrm{N}_{0}$ [1]. Ionospheric scintillation is typically modeled as an autoregressive (AR) process. In [13], the parameters are identified $a$ priori in an offline procedure, the measurement noise covariance is online adapted based on an $\mathrm{C} / \mathrm{N}_{0}$ estimator and a discriminator-based Kalman filter PLL structure estimating the scintillation phase is employed. In [14], an extended Kalman filter PLL structure with two outputs related to the inphase/quadrature (I/Q) branches and estimation of both scintillation amplitude and phase is employed, with the parameters of the AR model identified offline and adaptive measurement noise covariance update. This concept is generalized in [15] with an extended Kalman filter with increased complexity including online identification of the AR model parameters and online updating of the process noise covariance matrix based on the identification statistics. Other techniques also consider adaptive Kalman filtering with online adaptive estimation of the AR scintillation model parameter, as in [16], where a dual Kalman filter was applied. The scintillation phase and amplitude are estimated by a first Kalman filter. They are then used as measurements in the second Kalman filter to estimate the parameters of the respective AR process. Afterward, the LOS and scintillation phase error estimates are then fed back into the carrier signal replica generator by a linear quadratic Gaussian control approach. Especially for ionospheric monitoring, a (dual) Kalman smoother can be applied to further improve the estimates of scintillation amplitude and phase [17].

In this work, we explore scintillation phase and amplitude estimation for scintillation monitoring and mitigation. First, we consider carrier tracking loop structures for LOS tracking only, i.e., structures that do not explicitly account for the scintillation induced effects, but rather are designed for tracking the combination of LOS dynamics and scintillation induced variations present in the incoming signal and could be easily implemented in a state-of-the-art receiver of a monitoring station performing carrier synchronization. We consider a traditional FLL, which is composed of elements such as a linear loop filter, a numerically controlled oscillator (NCO), and a frequency discriminator, as well as a discriminator-based Kalman FLL. To perform ionospheric monitoring, we exploit the frequency content characteristics of both the traditional and the Kalman FLL based on the linear approximation of their tracking loops to propose an algorithm for estimating the phase variations induced by scintillation. The algorithm is composed of linear filtering of prompt I/Q correlator data of a FLL with a noncoherent delay locked loop (DLL) and loop filter observables of the FLL. It is designed to recover the complementary information in the frequency domain based on the sensitivity and complementary sensitivity transfer functions of the respective closed-loop linear approximation. It can easily be either added to an already implemented traditional or Kalman FLL in a receiver for online estimation to feed a scintillation monitor or can be employed in data postprocessing if the observables are stored. The filters' parameters can be adjusted to delimit the frequency band of interest, as is typically done in batch post-processing with Butterworth filters [18].

Secondly, for ionospheric scintillation monitoring and mitigation, we propose two Kalman filter PLLs for carrier synchronization with increased robustness to scintillation induced effects: a discriminator-based Kalman PLL and an extended Kalman PLL. The discriminator-based Kalman PLL model includes the dynamics of the scintillation phase and the extended Kalman PLL model includes the dynamics of scintillation phase and amplitude, in addition to the LOS dynamics in both cases. Scintillation phase and amplitude are modeled by a suitable kinematic process model [19], and are considered independent to the LOS dynamics, which are also modeled by a kinematic process model. Compared to the AR process model, this process model allows a simpler formulation, since parameter identification is not required. Furthermore, the kinematic consistency among its state variables allows to naturally impose constraints in the filter iterations to prevent a negative scintillation amplitude estimate with corresponding half-cycle phase shift estimate in the extended Kalman filter formulation.

We assess the performance of the proposed algorithms for scintillation monitoring and mitigation via numerical simulations by adding synthetic severe scintillation variations generated by the Cornell Scintillation Model (CSM) [20] to simulated baseband (BB) GPS L1 C/A code signal input data and comparing the algorithms' results regarding the estimation of scintillation induced effects and the LOS tracking to the results of state-of-the-art adaptive extended Kalman PLL with online parameter identification of the scintillation AR process model as defined in [15].

\section{SignAL MODEL}

Disregarding the navigation message, a GNSS BB signal received from one satellite with bandwidth $B$ can be given by its $\mathrm{I} / \mathrm{Q}$ components

$$
\left\{\begin{array}{l}
I(t)=A(t) c(t-\tau(t)) \cos \left(\phi_{T}(t)\right)+n_{I}(t) \\
Q(t)=A(t) c(t-\tau(t)) \sin \left(\phi_{T}(t)\right)+n_{Q}(t),
\end{array}\right.
$$

where $A(t)$ is the signal amplitude, $c(t-\tau(t))$ is a binary pseudorandom sequence delayed by $\tau(t)$, and $\phi_{T}(t)$ is the total carrier phase, including Doppler frequency shift, reference oscillator offset, and other sources of phase variations, such as ionospheric scintillation. So the complex BB signal is

$$
s(t)=I(t)+j Q(t),
$$

and $n_{I}(t) \in \mathbb{R}$ and $n_{Q}(t) \in \mathbb{R}$ are the independent noise processes of the complex Gaussian white noise

$$
n(t)=n_{I}(t)+j n_{Q}(t),
$$

each with power spectral density $N_{0} / 2$. Considering only a scintillation induced term multiplicative in amplitude and 
additive in phase $\rho(t) e^{j \phi(t)}$ and a LOS amplitude $A_{0}(t)$, the signal amplitude can be written as

$$
A(t)=A_{0}(t) \rho(t)
$$

and the signal total phase as

$$
\phi_{T}(t)=\phi_{D}(t)+\phi(t)
$$

where $\phi_{D}(t)$ is the phase related to the Doppler frequency shift while disregarding other sources of phase variations. The phase $\phi_{D}(t)$ is the integral of the angular Doppler frequency $f_{D}(t)$

$$
\phi_{D}(t)=\int_{t_{0}}^{t} f_{D}(\tilde{t}) d \tilde{t}
$$

which can be approximated by a Taylor series in a sufficiently short period of time in relation to the LOS dynamics. Truncation of the series after the second order term can represent constant Doppler frequency drift in the LOS signal, and yields

$$
\phi_{D}(t)=\phi_{D}\left(t_{0}\right)+f_{D}\left(t_{0}\right)\left(t-t_{0}\right)+(1 / 2) a_{D}\left(t_{0}\right)\left(t-t_{0}\right)^{2},
$$

where $a_{D}(t)$ is the Doppler frequency drift. The complex BB signal (2) sampled with sampling frequency $f_{s}=\frac{1}{T_{s}}=2 B$, $n=0, \ldots, N-1$, and $k=0,1,2, \ldots, \in \mathbb{Z}^{+}$can be given as

$$
\begin{aligned}
s\left((k N+n) T_{s}\right)= & A\left((k N+n) T_{s}\right) c\left((k N+n) T_{s}-\tau\left(n T_{s}\right)\right) \\
& e^{j \phi_{T}\left((k N+n) T_{s}\right)}+n\left((k N+n) T_{s}\right),
\end{aligned}
$$

where $N=f_{s} T_{I}$ samples are collected for the $k$-th coherent integration period of duration $T_{I}$. Assuming that $A(t), \phi(t)$, $f_{D}(t)$, and $\tau(t)$ are approximately constant over one coherent integration period, the discrete input sequence can be written in vector form as

$$
\mathbf{s}[k]=A[k] e^{j \phi[k]}\left(\mathbf{c}[k ; \tau[k]] \odot \mathbf{d}\left[k ; f_{D}[k]\right]\right)+\mathbf{n}[k],
$$

where

$$
\begin{aligned}
\mathbf{s}[k]= & {\left[s\left(k N T_{s}\right), \ldots, s\left((k N+N-1) T_{s}\right)\right]^{T} } \\
\mathbf{n}[k]= & {\left[n\left(k N T_{s}\right), \ldots, n\left((k N+N-1) T_{s}\right)\right]^{T} } \\
\mathbf{c}[k ; \tau[k]]= & {\left[c\left(k N T_{s}-\tau[k]\right),\right.} \\
& \left.\ldots, c\left((k N+N-1) T_{s}-\tau[k]\right)\right]^{T} \\
\mathbf{d}\left[k ; f_{D}[k]\right]= & {\left[e^{j\left(f_{D}[k] k N T_{s}+\phi_{D}[k]\right)},\right.} \\
& \left.\ldots, e^{j\left(f_{D}[k](k N+N-1) T_{s}+\phi_{D}[k]\right)}\right]^{T},
\end{aligned}
$$

and $\odot$ denotes element-by-element multiplication of two vectors (Hadamard-Schur product). In the same way, all internal tracking loop variables running at $f_{s}$ can also be represented as $N \times 1$ vectors.

The result of the prompt correlation with the code $\mathbf{c}[k ; \hat{\tau}[k]]$ generated by an estimate of the time-delay $\hat{\tau}[k]$ after mixing with the carrier $\mathbf{d}\left[k ; \hat{f}_{D}[k]\right]$ generated by a Doppler phase $\hat{\phi}_{D}[k]$ and frequency $\hat{f}_{D}[k]$ estimate can be given by

$$
y[k]=\frac{1}{N} \mathbf{s}^{\mathrm{H}}[k]\left(\mathbf{c}[k ; \hat{\tau}[k]] \odot \mathbf{d}\left[k ; \hat{f}_{D}[k]\right]\right),
$$

where ${ }^{\mathrm{H}}$ denotes Hermitian of a matrix or vector. Considering that $\tau[k] \approx \hat{\tau}[k]$ we get

$$
\begin{aligned}
y[k]=\frac{A[k]}{N} e^{-j\left(\phi[k]+\phi_{D}[k]-\hat{\phi}_{D}[k]\right)} & \\
& \sum_{n=0}^{N-1} e^{-j\left(f_{D}[k]-\hat{f}_{D}[k]\right) n T_{s}}+\eta_{D}[k] .
\end{aligned}
$$

The zero-mean complex white Gaussian noise $\eta_{D}[k]$ has variance $\sigma_{\eta_{D}}^{2}=\sigma_{n}^{2} / N$, where $\sigma_{n}^{2}=2 B N_{0}$ is the variance of $n\left((k N+n) T_{s}\right)$. With $\delta \phi_{D}[k]=\phi_{D}[k]-\hat{\phi}_{D}[k]$ and $\delta f_{D}[k]=f_{D}[k]-\hat{f}_{D}[k]$ we can derive an approximate expression for the prompt correlator output after mixing with the carrier generated by the receiver as ${ }^{1}$

$$
\begin{aligned}
& y[k]=A[k] \operatorname{sinc}\left(\frac{-\delta f_{D}[k] T_{s}}{2 \pi}\right) e^{-j\left(\phi[k]+\delta \phi_{D}[k]\right)} \\
& e^{-j\left[(N-1) T_{s} / 2\right] \delta f_{D}[k]}+\eta_{D}[k] .
\end{aligned}
$$

If $\left|\delta f_{D}[k] T_{s}\right| \ll 1, \operatorname{sinc}\left(\frac{-\delta f_{D}[k] T_{S}}{2 \pi}\right) \approx 1$. For an unitary amplitude of the carrier undisturbed by scintillation, $A[k]=$ $\rho[k]$ and $(N-1) T_{s} \approx T_{I}$, we can write the I/Q components of $y[k]=y_{I}[k]+j y_{Q}[k]$ as

$$
\left\{\begin{aligned}
y_{I}[k] & =\rho[k] \cos \left(-\phi[k]-\delta \phi_{D}[k]-\frac{T_{I}}{2} \delta f_{D}[k]\right) \\
& +\eta_{D_{I}}[k] \\
y_{Q}[k] & =\rho[k] \sin \left(-\phi[k]-\delta \phi_{D}[k]-\frac{T_{I}}{2} \delta f_{D}[k]\right) \\
& +\eta_{D_{Q}}[k],
\end{aligned}\right.
$$

with $\sigma_{\eta_{D_{I}}}^{2}=\sigma_{\eta_{D_{Q}}}^{2}=\sigma_{\eta_{D}}^{2} / 2$. Further simplifying by disregarding the term in $\delta f_{D}[k]$ leads to the nonlinear measurement equations commonly employed in the extended Kalman filter [14], [15] with

$$
\left\{\begin{aligned}
y_{I}[k] & =\rho[k] \cos \left(-\phi[k]-\delta \phi_{D}[k]\right)+\eta_{D_{I}}[k] \\
y_{Q}[k] & =\rho[k] \sin \left(-\phi[k]-\delta \phi_{D}[k]\right)+\eta_{D_{Q}}[k] .
\end{aligned}\right.
$$

Application of the four quadrant arctangent $\varepsilon_{P}[k]=$ $-\operatorname{atan} 2\left[y_{Q}[k], y_{I}[k]\right]$ yields

$$
\varepsilon_{P}[k]=\phi[k]+\delta \phi_{D}[k]+\eta_{A}[k],
$$

where $\eta_{A}[k]$ is the noise at the output of the four quadrant arctangent, with variance $\sigma_{\eta_{A}}^{2}$ that can be computed by approximate expression as shown in [21]. An estimation of the rate of $\varepsilon_{P}[k]$ can be obtained by the frequency discriminator [22]

$$
\varepsilon_{D}[k]=-\frac{\operatorname{atan} 2[\text { cross }, d o t]}{T_{I}}=\frac{\varepsilon_{P}[k]-\varepsilon_{P}[k-1]}{T_{I}}
$$

where cross $=y_{Q}[k] y_{I}[k-1]-y_{Q}[k-1] y_{I}[k]$ and $d o t=$ $y_{I}[k] y_{I}[k-1]+y_{Q}[k] y_{Q}[k-1]$.

${ }^{1} \sum_{n=0}^{N-1} e^{j n z}=e^{j(N-1) z / 2} \frac{\sin (N z / 2)}{\sin (z / 2)} \approx N e^{j(N-1) z / 2} \operatorname{sinc}\left[\frac{z}{2 \pi}\right]$, $\operatorname{sinc}(t)=\sin (\pi t) /(\pi t)$ and $z=-\delta f_{D}[k] T_{s}$. 


\section{LINEAR APPROXIMATION OF A TRADITIONAL FLL}

The term "traditional" is used here to refer to code, phase or frequency tracking loops implemented with loop filters and numeric controlled oscillators (NCO) in closed-loop. Their linear approximations are useful for the purposes of filter design and analysis, providing means to evaluate their behaviour in the frequency domain, once the nonlinear tracking loop is operating inside a region where the linear approximation is considered to be valid. A traditional FLL with a noncoherent DLL receiver is shown in Figure 1 , where $\mathbf{e}[k] \in \mathbb{R}^{N \times 1}$, $\mathbf{l}[k] \in \mathbb{R}^{N \times 1}$, and $\mathbf{p}[k] \in \mathbb{R}^{N \times 1}$ collect the sampled early, late, and prompt code replicas. The DLL and FLL include a NCO. Each correlator branch includes an integrate and dump (I\&D) block, and $\left[I_{e}[k], Q_{e}[k]\right]^{\mathrm{T}},\left[I_{l}[k], Q_{l}[k]\right]^{\mathrm{T}}$, and $\left[I_{p}[k], Q_{p}[k]\right]^{\mathrm{T}}$ are, respectively, the early, late, and prompt output I/Q samples at the $k$ th integration period.

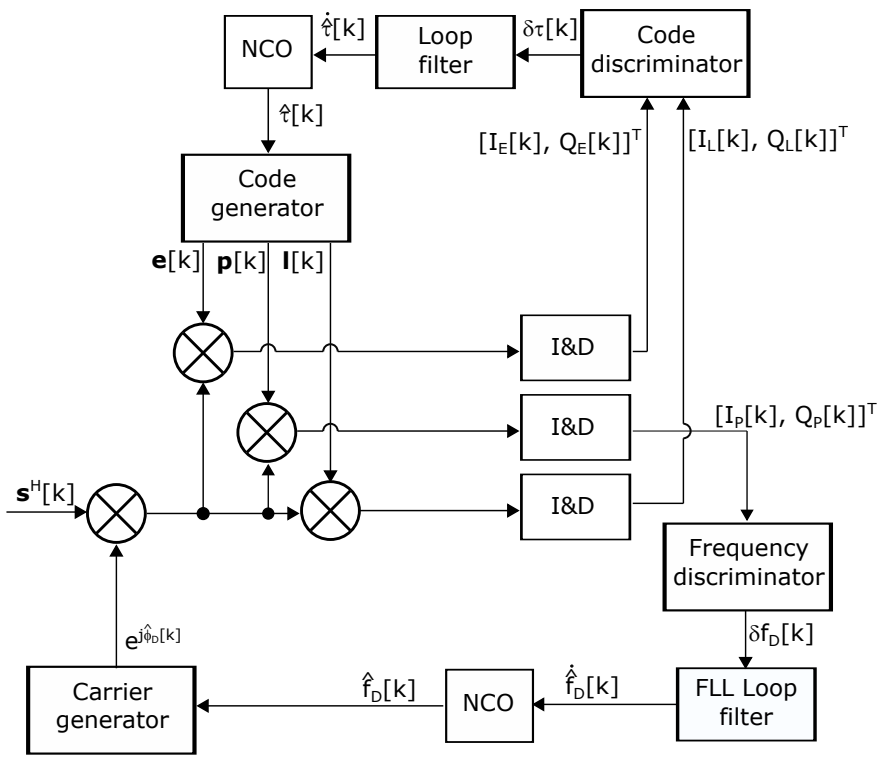

Fig. 1. Traditional FLL/DLL receiver.

Neglecting the coupling between the code and carrier loops, a linear approximation in the discrete-time domain for the FLL can be obtained by considering the NCO block as an integrator, and the operations of mixing the input signal with the generated carrier replica, correlation, and frequency discriminator computation as the difference $f_{D}[k]-\hat{f}_{D}[k]=\delta f_{D}[k]$. With these assumptions, we can establish the block diagram as shown in Figure 2 for the linear approximation of the FLL in the $\mathrm{Z}$ domain. Figure $2 \mathrm{a}$ shows the detailed block diagram. The frequency discriminator provides the Doppler shift error signal $\delta f_{D}(z)$ and $G_{F L L}(z)$ encompasses the loop filter and the NCO. The remaining integrator represents the replica carrier generation from the Doppler frequency shift estimate, as the loop is presented in terms of the total phase. A simplified block diagram in terms of Doppler frequency shift only can be obtained removing the derivative and integration blocks, as shown in Figure 2b. The loop filter transfer function, including the NCO integrator, is typically of second or third order [23],

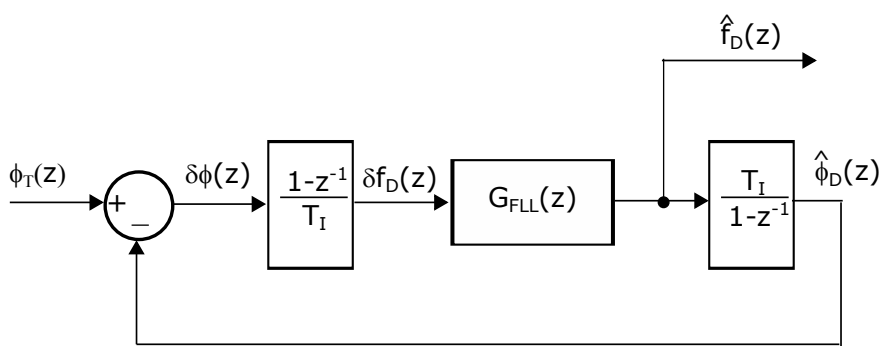

(a) Complete.

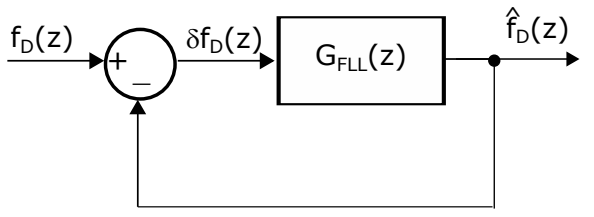

(b) Simplified.

Fig. 2. FLL linear approximation.

[24]. A second order loop filter has the following $\mathrm{Z}$ transform

$$
G_{F L L}(z)=\frac{\left(T_{I}^{2} \omega_{n}^{2}+2 \xi \omega_{n} T_{I}\right)-2 \xi \omega_{n} T_{I} z^{-1}}{1-2 z^{-1}+z^{-2}},
$$

with damping ratio $\xi$ and natural frequency $\omega_{n}$. Typically, $\xi=$ $1 / \sqrt{2}$ and $\omega_{n}$ is the design parameter. A third order loop filter has the following $\mathrm{Z}$ transform

$$
G_{F L L}(z)=\frac{\begin{array}{c}
\left.T_{I}^{3} \omega_{0}^{3}+a_{3} T_{I}^{2} \omega_{0}^{2}+b_{3} T_{I} \omega_{0}\right) \\
+\left(-a_{3} T_{I}^{2} \omega_{0}^{2}-2 b_{3} T_{I} \omega_{0}\right) z^{-1}+T_{I} b_{3} \omega_{0} z^{-2}
\end{array}}{1-3 z^{-1}+3 z^{-2}-z^{-3}},
$$

where typically $a_{3}=1.1$ and $b_{3}=2.4$ and $\omega_{0}$ is the design parameter. Working with a $2^{\text {nd }}$ order loop filter, the closed loop has the following transfer function for the Doppler frequency shift

$\frac{\hat{f}_{D}}{\phi_{T}}(z)=\frac{\left(T_{I} \omega_{n}^{2}+2 \xi \omega_{n}\right)+\left(-T_{I} \omega_{n}^{2}-4 \xi \omega_{n}\right) z^{-1}+2 \xi \omega_{n} z^{-2}}{\left(T_{I}^{2} \omega_{n}^{2}+2 \xi \omega_{n} T_{I}+1\right)+\left(-2 \xi \omega_{n} T_{I}-2\right) z^{-1}+z^{-2}}$.

The expanded block diagram of Figure $2 b$ is shown in Figure 3 , where the internal states of the closed loop are depicted explicitly. The output of the upstream integrator, the state $\hat{\dot{f}}_{D_{1}}(z)$, is an estimate of the Doppler frequency drift, but the zeros of its transfer function are different from the zeros of the transfer function of $\hat{\dot{f}}_{D}(z)$, which is given by the integral of $\hat{f}_{D}(z)$. The error signal in terms of phase in Figure $2 \mathrm{a}$ is

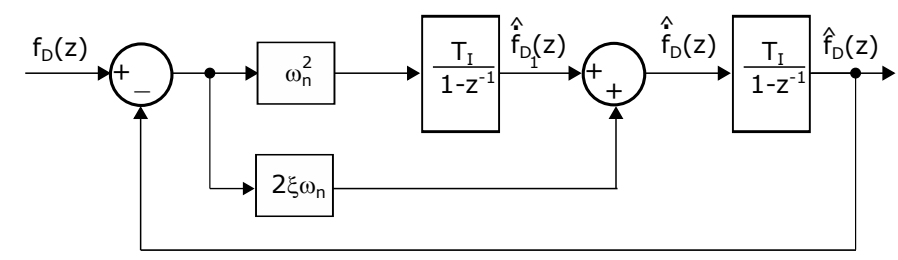

Fig. 3. Simplified FLL linear approximation expanded. 


$$
\begin{aligned}
\delta \phi(z) & =\phi_{T}(z)-\hat{\phi}_{D}(z) \\
& =\phi_{T}(z)-\frac{T_{I}}{1-z^{-1}} \hat{f}_{D}(z) \\
& =\left(1-\frac{T_{I}}{1-z^{-1}} \frac{\hat{f}_{D}}{\phi_{T}}(z)\right) \phi_{T}(z) .
\end{aligned}
$$

Therefore, for the $2^{\text {nd }}$ order loop filter, the transfer function for $\delta \phi(z)$ is

$$
\begin{aligned}
\frac{\delta \phi}{\phi_{T}}(z) & =\left(1-\frac{T_{I}}{1-z^{-1}} \frac{\hat{f}_{D}}{\phi_{T}}(z)\right) \\
& =\frac{1-2 z^{-1}+z^{-2}}{\left(T_{I}^{2} \omega_{n}^{2}+2 \xi \omega_{n} T_{I}+1\right)+\left(-2 \xi \omega_{n} T_{I}-2\right) z^{-1}+z^{-2}} .
\end{aligned}
$$

This phase error has information content in the frequency domain that is complementary to the integral of the Doppler frequency shift estimate $\hat{f}_{D}(z)$. This can be verified by adding the integral of (23) to (27), yielding

$$
\frac{\hat{f}_{D}(z) T_{I} /\left(1-z^{-1}\right)+\delta \phi(z)}{\phi_{T}(z)}=1 .
$$

This property also holds for the double integral of $\hat{\dot{f}}_{D}(z)$, but not for the double integral of $\hat{\dot{f}}_{D_{1}}(z)$.

\section{KALMAN FLL FOR LOS-ONLY TRACKING}

The state vector to be estimated by the Kalman filter is $\mathbf{x}[k] \in \mathbb{R}^{P \times 1}$. In the more general case of the extended Kalman filter, the state evolution and the measurement equations are formed by nonlinear functions according to the discrete system equations

$$
\begin{aligned}
& \mathbf{x}[k]=\mathbf{f}(\mathbf{x}[k-1])+\boldsymbol{\nu}[k] \\
& \mathbf{y}[k]=\mathbf{h}(\mathbf{x}[k])+\mathbf{n}[k],
\end{aligned}
$$

where $\mathbf{f} \in \mathbb{R}^{P \times 1}$ and $\mathbf{h} \in \mathbb{R}^{M \times 1}$ are nonlinear functions of the state vector $\mathbf{x}[k] \in \mathbb{R}^{P \times 1}, \mathbf{y}[k] \in \mathbb{R}^{M \times 1}$ is the measurement vector, $\boldsymbol{\nu}[k] \in \mathbb{R}^{P \times 1}$ and $\mathbf{n}[k] \in \mathbb{R}^{M \times 1}$ are independent Gaussian process and measurement noises whose covariance matrices are $\mathbf{Q} \in \mathbb{R}^{P \times P}$ and $\mathbf{R} \in \mathbb{R}^{M \times M}$, respectively. The propagation step and the update step of the Kalman filter can be given as

$$
\begin{aligned}
\hat{\mathbf{x}}[k \mid k-1] & =\mathbf{f}(\hat{\mathbf{x}}[k-1]) \\
\mathbf{P}[k \mid k-1] & =\mathbf{F}[k-1] \mathbf{P}[k-1] \mathbf{F}^{\mathrm{T}}[k-1]+\mathbf{Q}
\end{aligned}
$$

and

$$
\begin{aligned}
\mathbf{K}[k] & =\mathbf{P}[k \mid k-1] \mathbf{H}^{\mathrm{T}}[k]\left(\mathbf{H}[k] \mathbf{P}[k \mid k-1] \mathbf{H}^{\mathrm{T}}[k]+\mathbf{R}\right)^{-1} \\
\mathbf{P}[k] & =(\mathbf{I}-\mathbf{K}[k] \mathbf{H}[k]) \mathbf{P}[k \mid k-1] \\
\hat{\mathbf{x}}[k] & =\hat{\mathbf{x}}[k \mid k-1]+\mathbf{K}[k]\{\mathbf{y}[k]-\mathbf{h}(\hat{\mathbf{x}}[k \mid k-1])\} .
\end{aligned}
$$

The measurement residual or innovation is $\varepsilon[k]=\mathbf{y}[k]-$ $\mathbf{h}(\hat{\mathbf{x}}[k \mid k-1]), \mathbf{P}[k] \in \mathbb{R}^{P \times P}$ is the state covariance matrix and $\mathbf{K}[k] \in \mathbb{R}^{P \times M}$ is the Kalman gain. In the case of the Kalman filter, the process and measurement equations are linear functions of the state vector, so that $\mathbf{f}(\mathbf{x}[k-1])=$ $\mathbf{F x}[k-1]$ with $\mathbf{F} \in \mathbb{R}^{P \times P}$ and $\mathbf{h}(\mathbf{x}[k])=\mathbf{H x}[k]$ with $\mathbf{H} \in \mathbb{R}^{M \times P}$, respectively. In the case of the extended Kalman filter, $\mathbf{f}(\mathbf{x}[k-1])$ and/or $\mathbf{h}(\mathbf{x}[k])$ are nonlinear functions of the states, whose Jacobians

$$
\mathbf{F}[k-1]=\left.\frac{\partial \mathbf{f}}{\partial \mathbf{x}}\right|_{\mathbf{x}=\hat{\mathbf{x}}[k-1]}, \mathbf{H}[k]=\left.\frac{\partial \mathbf{h}}{\partial \mathbf{x}}\right|_{\mathbf{x}=\hat{\mathbf{x}}[k \mid k-1]}
$$

are employed in the prediction and update equations of the extended Kalman filter.

The Kalman FLL for LOS dynamics tracking employing the kinematic process model given in [19] with

$$
\mathbf{x}_{D}[k]=\left[\begin{array}{ll}
f_{D}[k] & a_{D}[k]
\end{array}\right]^{\mathrm{T}}
$$

has the following state transition and state covariance matrices

$$
\mathbf{F}_{D}=\left[\begin{array}{cc}
1 & T_{I} \\
0 & 1
\end{array}\right], \mathbf{Q}_{D}=\sigma_{a_{D}}^{2}\left[\begin{array}{cc}
T_{I}^{3} / 3 & T_{I}^{2} / 2 \\
T_{I}^{2} / 2 & T_{I}
\end{array}\right]
$$

after truncation of (6) at the second order term and with Gaussian acceleration variance $\sigma_{a_{D}}^{2}$.

Considering a discriminator-based Kalman filter, the innovations sequence is formed by (20), providing an estimation of frequency error. Therefore, the observation matrix for the LOS-only FLL is

$$
\mathbf{H}_{D}=\left[\begin{array}{ll}
1 & 0
\end{array}\right] .
$$

The block diagram of a receiver with a discriminator-based Kalman filter FLL and a traditional noncoherent DLL is shown in Figure 4.

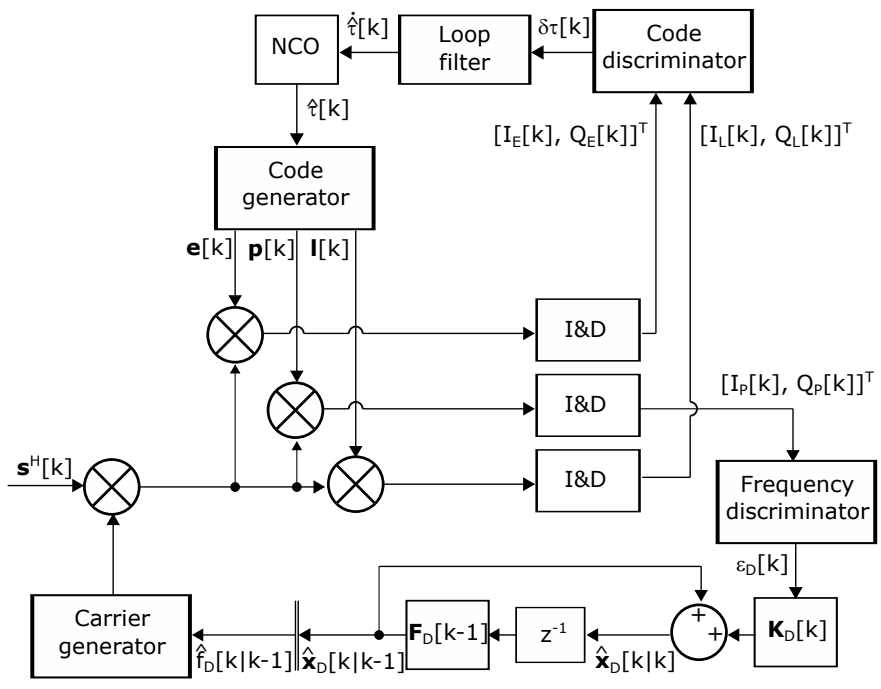

Fig. 4. Kalman FLL/traditional DLL receiver.

A linear approximation for the Kalman FLL of Figure 4 with gain

$$
\mathbf{K}_{D}[k]=\left[K_{D, 1}[k] \quad K_{D, 2}[k]\right]^{\mathrm{T}}
$$

can be obtained in the same manner as for the traditional FLL. The Kalman FLL has the same simplified representation of the FLL presented in Figure 3, substituting $2 \xi \omega_{n}$ by $K_{D, 1}[k]$ and $\omega_{n}^{2}$ by $K_{D, 2}[k]$. Continuous/discrete equivalence of Kalman filter equations can be found in [25], [26]. The difference between both second order filters is that the traditional FLL filter has fixed parameters while the parameters of the Kalman FLL are time-varying and computed according to the optimality premises of the Kalman filter. The state estimates 
$\hat{f}_{D}[k]=\hat{f}_{D}\left(k T_{I}\right)$ and $\hat{a}_{D}[k]=\hat{a}_{D}\left(k T_{I}\right)$ of the Kalman FLL are equivalent to $\hat{f}_{D}\left(k T_{I}\right)$ and $\hat{\dot{f}}_{D_{1}}\left(k T_{I}\right)$ of the linear approximation, respectively. The discrete equivalent estimate for $\hat{\dot{f}}_{D}\left(k T_{I}\right)$ in the Kalman filter is, correcting for the update rate $T_{I}$ for continuous/discrete equivalence [25]:

$$
\hat{\dot{f}}_{D}[k]=\hat{a}_{D}[k]+K_{D, 1}[k] \varepsilon_{D}[k] / T_{I} .
$$

\section{Scintillation Monitoring Based on LOS-ONLY FLLS}

The structure of the proposed signal processing algorithm for scintillation monitoring is based on the LOS dynamics tracking loops presented in Figures 1 and 4 for the traditional and Kalman FLL, respectively. Both tracking loops do not separate the part of the phase related to the LOS dynamics and the part induced by ionospheric scintillation of the incoming signals, performing carrier synchronization of the total phase. Surely, there are differences between traditional and Kalman filter tracking loops in terms of robustness to scintillation [12], [27], but regardless of these differences, our proposed algorithm for scintillation monitoring exploits both loops equivalently to provide estimates of the scintillation phase.

The proposed scintillation monitoring algorithm does not interfere in the existing traditional FLL or Kalman FLL designed for LOS tracking in a receiver, regardless of the design parameters of the filters. Once the I/Q outputs from the prompt correlator and the Doppler frequency shift estimate are available or can be derived from other available observables, the algorithm can be employed for offline post-processing of the data or can be implemented in the receiver without changing its current structure, just appending the signal processing to provide online scintillation estimates. This is the case for ionospheric monitoring receivers, that provide estimates of LOS phase evolution based on a known static receiver position and, typically, with a very precise reference oscillator.

Application of a phase discriminator on $\left[I_{P}[k], Q_{P}[k]\right]^{\mathrm{T}}$ provides a signal whose linear approximation in discrete domain is $\delta \phi[k]$ in Figure 2a, which is the integral of the frequency error $\delta f_{D}[k]$ in Figure 2, computed by the frequency discriminator of the FLL. Typically, the phase discriminator employed in phase tracking loops is the two-quadrant arc tangent (Costas loop), that would generate $\delta \phi[k]=$ $-\operatorname{atan}\left[Q_{P}[k] / I_{P}[k]\right]$ in the interval $[-\pi / 2, \pi / 2]$. Instead, we consider the total scintillation phase variation in the full circle $[-\pi, \pi]$, generating $\delta \phi[k]=-\operatorname{atan} 2\left[Q_{P}[k], I_{P}[k]\right]$. In the problem formulation, from (1) on, we discard the navigation message bits of the received signal. Therefore, we consider the existence of a navigation bit transition detector in the receiver to avoid half cycle spurious transitions in $\delta \phi[k]$.

Considering constant uncertain Doppler frequency drift, the LOS dynamics can be suitably approximated by the series expansion truncated at the $2^{\text {nd }}$ order term in (7). This is the case, for example, for static receivers on ground. In the traditional or Kalman FLL, $\hat{\dot{f}}_{D}[k]$ becomes biased, which, for the traditional FLL, is the loop filter output just prior to the $\mathrm{NCO}$ in Figure 1 or which can be derived for the Kalman
FLL according to (37). Since the FLL is closing the loop in frequency, $\delta \phi[k]$ may also become biased in the initial transient.

Our proposed scintillation monitor takes the complementary information from $\delta \phi[k]$ and $\hat{\dot{f}}_{D}[k]$ by adding the high frequency content in $\delta \phi[k]$ to the low frequency content of the double integral approximation of $\hat{\dot{f}}_{D}[k]$, handling the biases built up and the double integral divergence due to small errors related to nonlinearities, noise, and other sources by only considering the frequency band of interest.

\section{A. Traditional FLL}

The scintillation monitor with a traditional FLL for phase variations recovery is illustrated in Figure 5. In this figure, the blocks with solid lines describe the traditional FLL from Figure 1 while the blocks with dashed lines describe the scintillation monitor, that uses $\left[I_{P}[k], Q_{P}[k]\right]^{\mathrm{T}}$ from the prompt correlator and $\hat{\dot{f}}_{D}[k]$ from the loop filter. The filters in the dashed blocks are presented in their $\mathrm{Z}$ transform.

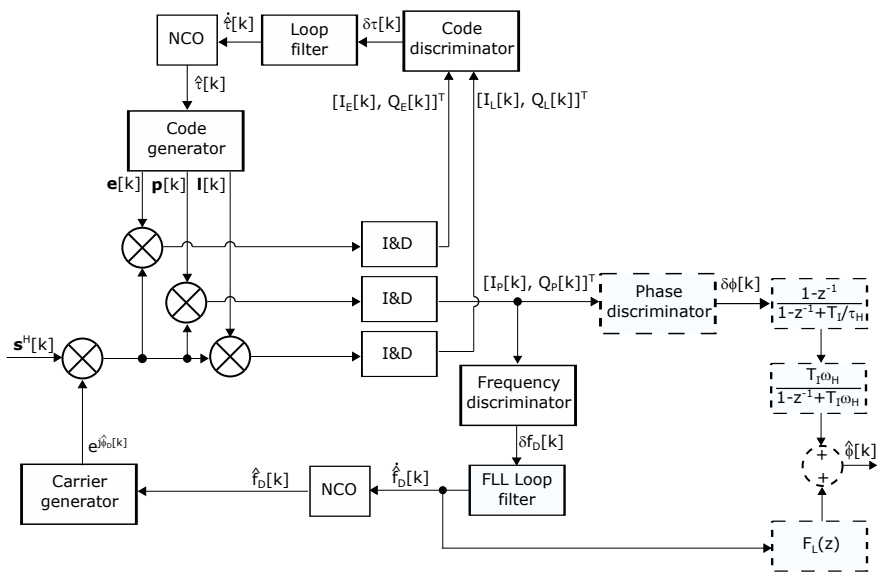

Fig. 5. Traditional FLL/DLL receiver with scintillation phase estimation.

The FLL tracks the input signal up to its bandwidth, so $\delta \phi[k]$ coming from the atan 2 discriminator has a frequency content above the FLL bandwidth, added to a bias resulting from initial filter transient. The task of the first filter applied to $\delta \phi[k]$ is to wash-out the bias, and the second filter is a roll-off filter with the task of limiting the higher frequency content. The Doppler frequency drift estimate, which has the low frequency content of the FLL bandwidth, is a second derivative of the phase. Added to the closed-loop dynamics there is also the Doppler frequency drift bias, slowly varying, dependent on the satellite pass. The purpose of the filter $F_{L}(z)$ is, therefore, to approximate the second integral while filtering out the slowly varying bias caused by nonlinearities and other sources. $F_{L}(z)$, as shown in Figure 6, is a $3^{\text {rd }}$ order high-pass filter in $u(z)$ to $y(z)$, where we can set $a_{3}=1.1$ and $b_{3}=2.4$.

The filter $F_{L}(z)$ is shown in the $\mathrm{Z}$ domain. All the filters can be implemented by taking their $\mathrm{Z}$ transform or, as typically done, by taking a discrete approximation of their integrators [23]. The frequency limits of the scintillation monitor are defined by $\omega_{L}$ for the lowest frequency and $\omega_{H}$ for the highest frequency. 


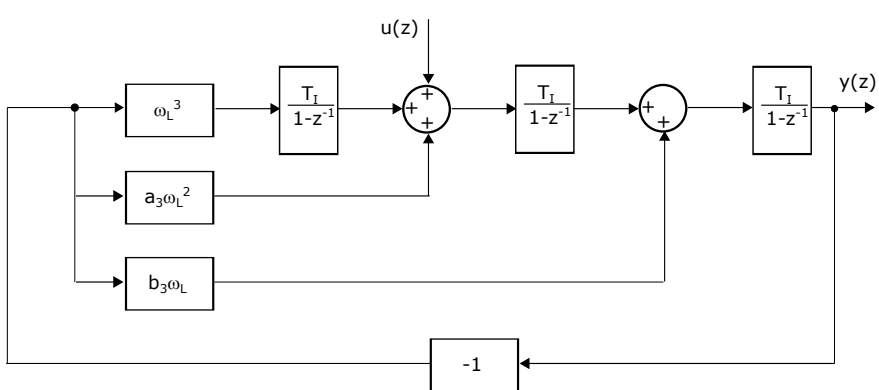

Fig. 6. Filter $F_{L}(z)$.

\section{B. Kalman FLL}

The filters employed in the scintillation monitor with a Kalman FLL are the same filters employed in the scintillation monitor with a traditional FLL. Naturally, the tuning parameters $\tau_{H}, \omega_{H}$ and $\omega_{L}$ may be adjusted to different values. The only difference in the signal processing is that $\hat{\dot{f}}_{D}[k]$ must be derived as (37) from the Kalman FLL to have complementary frequency content, since the state $a_{D}[k]$ in the Kalman FLL, related to the Doppler frequency drift, is dynamically equivalent to $\hat{\dot{f}}_{D_{1}}$. The Kalman FLL with the scintillation monitoring signal processing is shown in Figure 7.

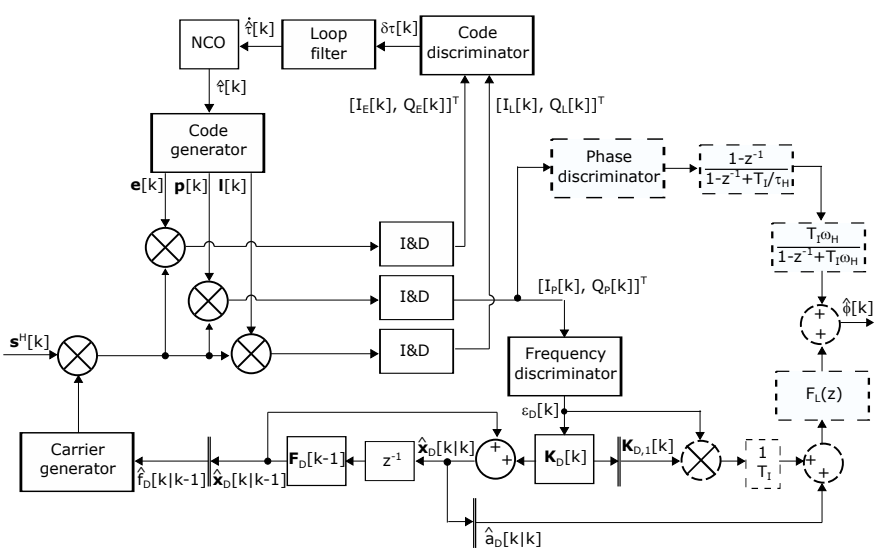

Fig. 7. Kalman FLL/traditional DLL receiver with scintillation phase estimation.

\section{Scintillation Mitigation With a Kalman PLL}

Let the LOS dynamics and scintillation induced phase and amplitude be considered independent processes, and both LOS and scintillation are modeled by the kinematic process equations. Then the scintillation process model can be appended to the LOS process model, so that LOS robust tracking and scintillation phase and amplitude estimation are accomplished by a Kalman filter. Hence, we model the LOS, scintillation phase, and scintillation amplitude as third order Wiener processes. Therefore, for scintillation mitigation purposes we consider a
Kalman PLL, whose LOS dynamics state-space equation can be given as

$$
\underbrace{\left[\begin{array}{c}
\phi_{D}[k] \\
f_{D}[k] \\
a_{D}[k]
\end{array}\right]}_{=\mathbf{x}_{D}[k]}=\underbrace{\left[\begin{array}{ccc}
1 & T_{I} & T_{I}^{2} / 2 \\
0 & 1 & T_{I} \\
0 & 0 & 1
\end{array}\right]}_{=\mathbf{F}_{D}} \underbrace{\left[\begin{array}{c}
\phi_{D}[k-1] \\
f_{D}[k-1] \\
a_{D}[k-1]
\end{array}\right]}_{=\mathbf{x}_{D}[k-1]}+\boldsymbol{\nu}_{D}[k],
$$

the scintillation phase process model is

$$
\underbrace{\left[\begin{array}{c}
\phi[k] \\
\dot{\phi}[k] \\
\ddot{\phi}[k]
\end{array}\right]}_{=\mathbf{x}_{\phi_{S}}[k]}=\underbrace{\left[\begin{array}{ccc}
1 & T_{I} & T_{I}^{2} / 2 \\
0 & 1 & T_{I} \\
0 & 0 & 1
\end{array}\right]}_{=\mathbf{F}_{\phi_{S}}} \underbrace{\left[\begin{array}{c}
\phi[k-1] \\
\dot{\phi}[k-1] \\
\ddot{\phi}[k-1]
\end{array}\right]}_{\mathbf{x}_{\phi_{S}}[k-1]}+\boldsymbol{\nu}_{\phi_{S}}[k],
$$

and the scintillation amplitude process model is

$$
\underbrace{\left[\begin{array}{c}
\rho[k] \\
\dot{\rho}[k] \\
\ddot{\rho}[k]
\end{array}\right]}_{=\mathbf{x}_{\rho_{S}}[k]}=\underbrace{\left[\begin{array}{ccc}
1 & T_{I} & T_{I}^{2} / 2 \\
0 & 1 & T_{I} \\
0 & 0 & 1
\end{array}\right]}_{=\mathbf{F} \rho_{S}} \underbrace{\left[\begin{array}{c}
\rho[k-1] \\
\dot{\rho}[k-1] \\
\ddot{\rho}[k-1]
\end{array}\right]}_{\mathbf{x}_{\rho_{S}}[k-1]}+\boldsymbol{\nu}_{\rho_{S}}[k] .
$$

So the LOS state vector is formed by Doppler phase $\phi_{D}[k]$, frequency shift $f_{D}[k]$ and frequency drift $a_{D}[k]$. The scintillation phase state vector is formed by phase $\phi[k]$, phase velocity $\dot{\phi}[k]$ and phase acceleration $\ddot{\phi}[k]$ and the scintillation amplitude state vector is formed by amplitude $\rho[k]$, amplitude velocity $\dot{\rho}[k]$ and amplitude acceleration $\ddot{\rho}[k]$. The process noises $\boldsymbol{\nu}_{D}[k], \boldsymbol{\nu}_{\phi_{S}}[k]$, and $\boldsymbol{\nu}_{\rho_{S}}[k]$ are defined by the covariance matrices $\mathbf{Q}_{D}=\sigma_{a_{D}}^{2} \mathbf{Q}_{I}, \mathbf{Q}_{\phi_{S}}=\sigma_{\ddot{\phi}_{S}}^{2} \mathbf{Q}_{I}$ and $\mathbf{Q}_{\rho_{S}}=\sigma_{\ddot{\rho}_{S}}^{2} \mathbf{Q}_{I}$, respectively, where

$$
\mathbf{Q}_{I}=\left[\begin{array}{ccc}
T_{I}^{5} / 20 & T_{I}^{4} / 8 & T_{I}^{3} / 6 \\
T_{I}^{4} / 8 & T_{I}^{3} / 3 & T_{I}^{2} / 2 \\
T_{I}^{3} / 6 & T_{I}^{2} / 2 & T_{I}
\end{array}\right]
$$

The process noise covariance matrix $\mathbf{Q}_{D}$ for the LOS dynamics is typically defined by $\sigma_{a_{D}}^{2}=0.2 \mathrm{rad}^{2} / \mathrm{s}^{5}$ [16]. The variances $\sigma_{\ddot{\phi}_{S}}^{2}$ and $\sigma_{\ddot{\rho}_{S}}^{2}$ are experimentally adjusted based on the analysis of the data. We explore two formulations of the Kalman PLL employing the proposed kinematic models for the scintillation processes: the first one is discriminatorbased, where the innovations are computed directly by the phase discriminator. In this case, the scintillation amplitude is not part of the state vector now given as

$$
\mathbf{x}_{1}[k]=\left[\begin{array}{ll}
\mathbf{x}_{D}^{\mathrm{T}}[k] & \mathbf{x}_{\phi_{S}}^{\mathrm{T}}[k]
\end{array}\right]^{\mathrm{T}} .
$$

The second one is an extended Kalman filter, with the complete state vector

$$
\mathbf{x}_{2}[k]=\left[\begin{array}{lll}
\mathbf{x}_{D}^{\mathrm{T}}[k] & \mathbf{x}_{\phi_{S}}^{\mathrm{T}}[k] & \mathbf{x}_{\rho_{S}}^{\mathrm{T}}[k]
\end{array}\right]^{\mathrm{T}} .
$$

The state transition matrices $\mathbf{F}_{1}$ and $\mathbf{F}_{2}$ for, respectively, $\mathbf{x}_{1}$ and $\mathbf{x}_{2}$, are

$$
\mathbf{F}_{1}=\left[\begin{array}{cc}
\mathbf{F}_{D} & \mathbf{0} \\
\mathbf{0} & \mathbf{F}_{\phi_{S}}
\end{array}\right], \quad \mathbf{F}_{2}=\left[\begin{array}{ccc}
\mathbf{F}_{D} & \mathbf{0} & \mathbf{0} \\
\mathbf{0} & \mathbf{F}_{\phi_{S}} & \mathbf{0} \\
\mathbf{0} & \mathbf{0} & \mathbf{F}_{\rho_{S}}
\end{array}\right]
$$

with the respective covariance matrices

$$
\mathbf{Q}_{1}=\left[\begin{array}{cc}
\mathbf{Q}_{D} & \mathbf{0} \\
\mathbf{0} & \mathbf{Q}_{\phi_{S}}
\end{array}\right], \quad \mathbf{Q}_{2}=\left[\begin{array}{ccc}
\mathbf{Q}_{D} & \mathbf{0} & \mathbf{0} \\
\mathbf{0} & \mathbf{Q}_{\phi_{S}} & \mathbf{0} \\
\mathbf{0} & \mathbf{0} & \mathbf{Q}_{\rho_{S}}
\end{array}\right]
$$


In the discriminator-based Kalman filter, the innovations are directly computed by (19), so that

$$
\mathbf{H}_{1}=\left[\begin{array}{llllll}
1 & 0 & 0 & 1 & 0 & 0
\end{array}\right] \text {. }
$$

In case of the extended Kalman filter, we have (18) as the nonlinear observation equations, whose linearization leads to

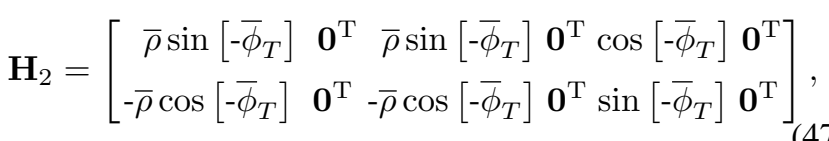

where $\bar{\rho}=\rho[k \mid k-1]$ and $\bar{\phi}_{T}=\phi[k \mid k-1]+\phi_{D}[k \mid k-$ $1]$, from the prediction step of the Kalman filter, and $\mathbf{0}=$ $\left[\begin{array}{ll}0 & 0\end{array}\right]^{\mathrm{T}}$. The measurement noise covariance matrix for the discriminator-based filter is defined by the atan 2 discriminator noise approximation [21]

$$
\mathbf{R}_{1}=\frac{1}{2 c / n_{0} T_{I}}\left(1+\frac{1}{c / n_{0} T_{I}}\right)
$$

where $c / n_{0}$ is the carrier to noise ratio in $\mathrm{Hz}$, obtained from $C / N_{0}$ in $\mathrm{dB}-\mathrm{Hz}$ as $c / n_{0}=10^{C / N_{0} / 10}$. We define $\mathbf{R}_{1}$ by fixing the value of the carrier to noise ratio at the relatively low value of $C / N_{0}=25 \mathrm{~dB}-\mathrm{Hz}$, so the covariance is adjusted for weaker signals but is high enough to cover stronger signals above this level. The discriminator-based filter is chosen to be non-adaptive so we can have a simpler model, to be compared to the more complex adaptive filters. The measurement noise covariance matrix $\mathbf{R}_{2}$ for the extended Kalman filter is adaptively adjusted based on $C / N_{0}$ estimation, according to [14].

\section{A. Constraining $\rho[k]$ to positive values in the extended Kalman filter}

For the extended Kalman filter the estimates of the scintillation amplitude can become negative due to abrupt transients in the input signal, resulting in disturbing transients and possibly in divergence of the extended Kalman filter. This occurs very often in the case of deep amplitude fades occurring during severe scintillation scenarios, depending on the level of existing abrupt transients in the input signal and also on the defined process and measurement noises covariance matrices of the Kalman filter. This loss of consistency can impair the accuracy of scintillation amplitude and phase estimates and their mitigation in the extended Kalman filter tracking loop.

The adaptive tuning of the process noise covariance matrix based on the parameter identification results of the scintillation $\mathrm{AR}$ processes and the measurement noise covariance matrix based on the $C / N_{0}$ estimator [15] can improve the filter robustness in this regard, but not prevent the occurrence in all cases, depending on the level of the transients in the input signal.

The introduction of a kinematic model for both scintillation phase and amplitude processes provides a natural form to exploit the kinematic relation between the variables to naturally constrain the extended Kalman filter to provide positive amplitude estimates at each iteration, keeping consistency between scintillation amplitude and phase estimates. Considering a complex variable $P(t)=G(t) e^{j \theta(t)} \in \mathbb{C}$, changing the value of $G(t) \in \mathbb{R}$ from positive to negative is equivalent to changing $\theta(t) \in \mathbb{R}$ to $\theta(t) \pm \pi$ and keeping $G(t)$ positive in order to have the same value of $P(t)$. Thus, we constrain the extended Kalman filter scintillation amplitude and phase estimates by

$$
\hat{\rho}[k \mid k-1]=\left\{\begin{aligned}
-\hat{\rho}[k \mid k-1], & \text { for } \hat{\rho}[k \mid k-1]<0 \\
\hat{\rho}[k \mid k-1], & \text { else }
\end{aligned}\right.
$$

and

$$
\hat{\phi}[k \mid k-1]=\left\{\begin{array}{ll}
\hat{\phi}[k \mid k-1]+\pi, & \text { for } \hat{\rho}[k \mid k-1]<0 \\
\hat{\phi}[k \mid k-1], & \text { else }
\end{array} .\right.
$$

Thus, the same predicted values by the nonlinear observation equations and the same $\mathbf{H}_{2}$, obtained from the Jacobian of those equations, can be used. To keep consistency, the kinematic model allows us to also invert $\hat{\dot{\rho}}[k \mid k-1], \hat{\ddot{\rho}}[k \mid k-1]$, $\hat{\dot{\phi}}[k \mid k-1]$ and $\hat{\ddot{\phi}}[k \mid k-1]$, if $\hat{\rho}[k \mid k-1]<0$.

\section{Evaluation of the Proposed Algorithms FOR SCINTILlation MONITORING AND Mitigation}

In this section the proposed algorithms for scintillation monitoring and mitigation are evaluated by numerical simulations considering synthetic scintillation input data generated by the CSM [20] in a severe scintillation scenario defined by amplitude strength index $\mathrm{S}_{4}=0.8$ and decorrelation time $\tau_{0}=0.1$. We consider a maximum Doppler frequency drift of $0.94 \mathrm{~Hz} / \mathrm{s}$ that an on-ground static receiver would experience based on the GPS satellites' orbital dynamics, added to the CSM scintillation variation data. In this work we consider GPS L1 C/A code BB signals with $B=2.046 \mathrm{MHz}$. No navigation bit transitions are considered and the carrier amplitude $A$ is unitary. The same realization of a Gaussian noise sequence is added to the sampled I/Q signal components in all simulations such that, for the receiver sampling frequency $f_{s}=2 B=$ $4.092 \mathrm{MHz}$ and an unitary carrier amplitude, results in the noise power $2 B N_{0}$ for $C / N_{0}=45 \mathrm{~dB}-\mathrm{Hz}$. We will consider receiving only one satellite signal with the pseudorandom noise sequence PRN $=1$. The early-late correlator spacing of the noncoherent DLL is $\Delta T_{c}=0.5$ chips and the coherent integration time is $T_{I}=1 \mathrm{~ms}$, integrating $N=f_{s} T_{I}=4092$ signal samples per cycle. Therefore, the same input signal including the same noise realization is considered for all simulated algorithms. The results are presented in terms of the root mean square error

$$
\operatorname{RMSE}_{x}[k]=\sqrt{\frac{1}{k} \sum_{i=1}^{k}(x[k]-\hat{x}[k])^{2}},
$$

with $x$ being substituted by the analyzed parameters. In the following we label the monitoring algorithm for scintillation phase recovery based on the traditional FLL as TFLL-REC, the monitoring algorithm for scintillation phase recovery based on the Kalman FLL as KFLL-REC, the mitigation algorithm based on the discriminator-based PLL and kinematic process models as KPLL-sKIN, and the mitigation algorithm based on the extended Kalman PLL with kinematic process models and adaptive measurement covariance matrix as EKPLL-sKIN$A D A P T$. As a benchmark algorithm we consider the adaptive 
extended Kalman PLL with AR scintillation model presented in [15] and we label this algorithm as EKPLL-sAR-ADAPT in the following.

A Kalman DLL would further reduce the noise sensitivity of the code loop [28], [29], but as we are only interested in the evaluation of the proposed algorithms with respect to carrier phase estimation, we use a second order noncoherent DLL configured with the initial time-delay estimate being the timedelay of the simulated input signal as well as with a very small loop bandwidth and extended correlators [28] for the early and late correlators in order to reduce the noise and keep the code replica synchronized with the input signal, so that approximations (18), (19) and (20) are valid. The DLL's second order loop filter is set with damping ratio $\xi=1 / \sqrt{2}$ and noise bandwidth $B_{n_{D L L}}=0.02 \mathrm{~Hz}$. The extended early and late correlators use 20 noncoherent correlations.

The traditional FLL of TFLL-REC employs a second order loop filter with damping ratio $\xi=1 / \sqrt{2}$, a noise bandwidth $B_{n_{F L L}}=2 \mathrm{~Hz}$, and the discriminator given in (20). The scintillation filters' parameters of TFLL-REC are tuned to $\tau_{H}=200 \mathrm{~s}, \omega_{H}=2 \pi \times 50 \mathrm{rad} / \mathrm{s}$ and $\omega_{L}=2 \pi \times 10^{-6} \mathrm{rad} / \mathrm{s}$, with $a_{3}=1.1$ and $b_{3}=2.4$. The Kalman FLL of KFLL-REC is defined by (34) and (35). The scintillation filters' parameters of KFLL-REC are tuned to $\tau_{H}=300 \mathrm{~s}, \omega_{H}=2 \pi \times 50 \mathrm{rad} / \mathrm{s}$, and $\omega_{L}=2 \pi \times 10^{-5} \mathrm{rad} / \mathrm{s}$ with $a_{3}=1.1$ and $b_{3}=2.4$.

The discriminator-based Kalman PLL of KPLL-SKIN is defined by $\mathbf{F}_{1}, \mathbf{Q}_{1}, \mathbf{H}_{1}$, and $\mathbf{R}_{1}$, as described in the previous section. The innovations are directly computed by the phase discriminator given in (19). The extended Kalman PLL of EKPLL-sKIN-ADAPT is defined by $\mathbf{F}_{2}, \mathbf{Q}_{2}, \mathbf{H}_{2}$, and $\mathbf{R}_{2}$ from the previous section, and the nonlinear observation equations are defined in (18).

The results of the proposed algorithms are compared to the results of the EKPLL-SAR-ADAPT with online parameter identification via recursive least squares [30] with 2000 points sliding window [31] following the approach presented in [15]. The RMSE of the scintillation phase $\phi[k]$, the scintillation amplitude $\rho[k]$, and the Doppler phase $\phi_{D}[k]$ for the different algorithms are presented in Figures 8, 9 and 10, respectively.

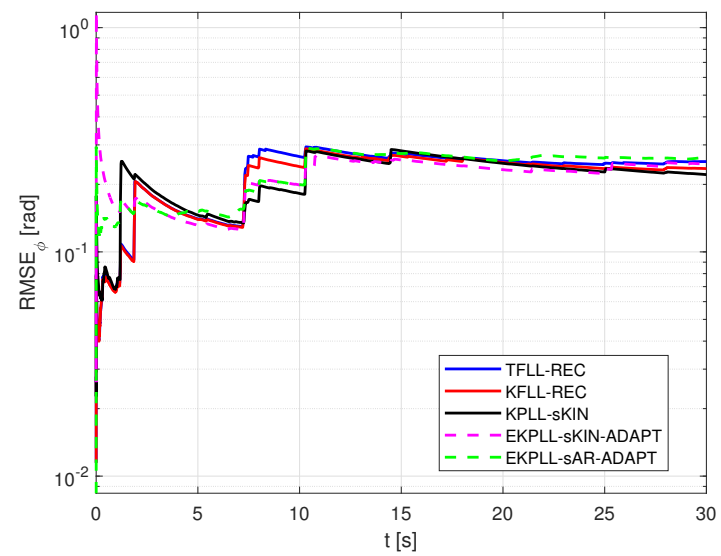

Fig. 8. Scintillation phase estimation $\mathrm{RMSE}_{\phi}$.

In Figure 8 we can observe that the $\mathrm{RMSE}_{\phi}$ for all algorithms is comparable. In Figure 9 we can see that as the extended Kalman filter formulations of EKPLL-SKIN-

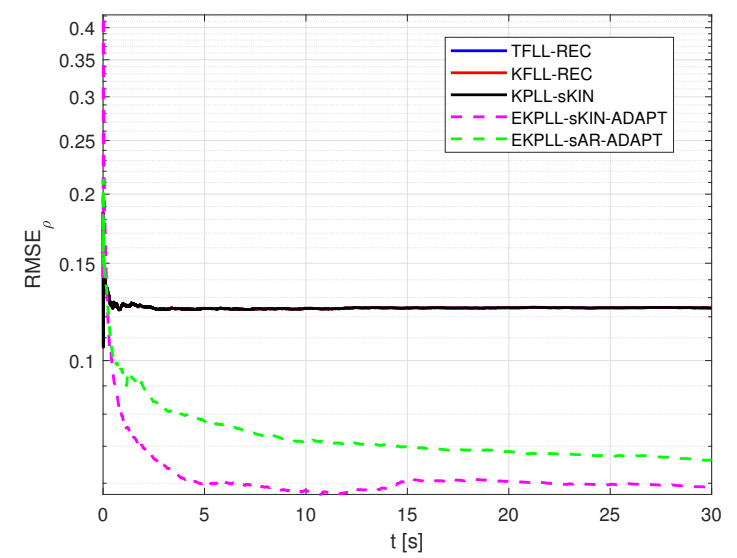

Fig. 9. Scintillation amplitude estimation $\operatorname{RMSE}_{\rho}$.

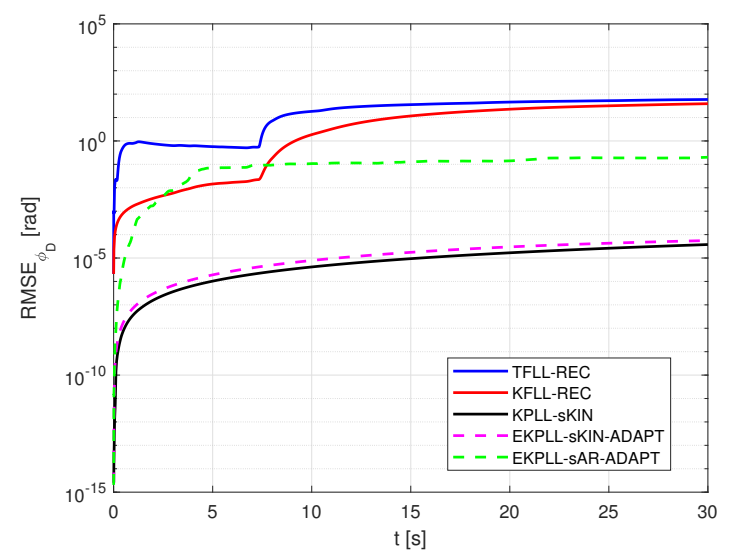

Fig. 10. Doppler phase estimation $\operatorname{RMSE}_{\phi_{D}}$.

$A D A P T$ and EKPLL-SAR-ADAPT include the scintillation amplitude in their processes models, they present a smaller $\operatorname{RMSE}_{\rho}$ compared to the other algorithms, with the $\operatorname{RMSE}_{\rho}$ of the EKPLL-sKIN-ADAPT being smaller than the of the benchmark algorithm EKPLL-sAR-ADAPT. The other algorithms are discriminator-based and thus their filters do not provide an amplitude estimate. The amplitude estimates for the discriminator-based algorithms are computed as $\hat{\rho}[k]=$ $\sqrt{I_{P}^{2}[k]+Q_{P}^{2}[k]}$. In Figure 9 their $\operatorname{RMSE}_{\rho}$ is superimposed by the black curve of the KPLL-sKIN.

Regarding scintillation mitigation, the $\mathrm{RMSE}_{\phi_{D}}$ of both the TFLL-REC and the KFLL-REC present the highest values, as shown in Figure 10, since the traditional and Kalman FLLs are LOS-only tracking loops. They are less robust to the scintillation effects since their formulation does not encompasses the scintillation dynamics in closed-loop. On the other hand, the Kalman PLLs perform better with respect to scintillation mitigation, with both the KPLL-sKIN and the EKPLL-SKIN$A D A P T$ presenting smaller $\mathrm{RMSE}_{\phi_{D}}$ when compared to the benchmark extended Kalman filter with online parameters identification called EKPLL-SAR-ADAPT.

A time series of scintillation phase and amplitude estimates is presented in Figure 11, for a time window around the deepest signal fade, with lowest peak of around $C / N_{0}=10 \mathrm{~dB}-\mathrm{Hz}$ occurring at $t=7.3 \mathrm{~s}$. The non adaptive algorithms present higher difference to the true scintillation phase when the signal strength is substantially reduced, in general. The estimated amplitudes are closer to the true scintillation amplitude, re- 
membering that the amplitude estimates of the discriminatorbased algorithms are computed as $\hat{\rho}[k]=\sqrt{I_{P}^{2}[k]+Q_{P}^{2}[k]}$, and are all superimposed by the black curve. The lowest amplitudes estimated of EKPLL-SKIN-ADAPT and EKPLL$s A R-A D A P T$ are presented in the data tip boxes, respectively $\hat{\rho}_{\mathrm{MIN}}=0.0005074$ and $\hat{\rho}_{\mathrm{MIN}}=-0.0204$, to illustrate the benefit of the constrained amplitude estimation by the EKPLL$s K I N-A D A P T$, where the amplitude is constrained to be positive. Depending on the level and duration, a negative amplitude estimate may lead to divergence of the extended Kalman filter, and possibly a fine tuning is required to provide a more conservative estimator.
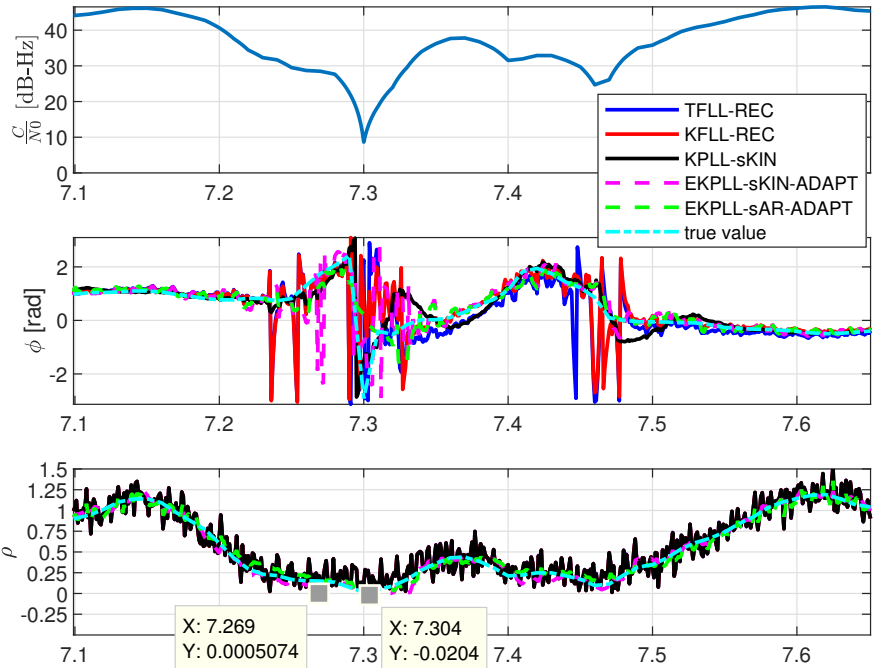

$\mathrm{t}[\mathrm{s}]$

Fig. 11. Scintillation phase and amplitude estimates time series in a deep fade.

\section{CONCLUSION}

We proposed a scintillation phase estimation algorithm based on linear filtering of observables with complementary frequency content with a traditional FLL and a Kalman filter FLL tracking the LOS dynamics only. This proposed algorithm can be used for online or offline scintillation monitoring. We also proposed a discriminator-based and an adaptive extended Kalman PLL, both with scintillation modeled using a suitable kinematic process model.

The proposed algorithms were evaluated via simulations with synthetic ionospheric scintillation data. The scintillation phase estimation results showed the capability of the monitoring algorithm to provide estimates with error comparable to the Kalman PLL structures employed in mitigation that explicitly model the scintillation induced effects. The proposed adaptive extended Kalman PLL presented smaller errors when compared to the considered benchmark adaptive extended Kalman PLL with online identification of the AR scintillation model [15]. Both the proposed Kalman PLL presented smaller error in LOS tracking than the benchmark approach given in [15], showing their capability of scintillation mitigation. Adding to this, their structures are simpler than of the benchmark algorithm, since parameter identification of the scintillation process model is not required and scintillation amplitude signal inversions can be naturally handled in the used extended formulation.
Since the monitoring algorithm is composed of stable linear filters running in open-loop with the FLL observables as inputs, its convergence depends on the LOS-only FLLs convergence. Once there is a loss-of-lock in the FLLs or a major disturbance, induced by ionospheric scintillation, the algorithm may produce permanent or temporary spurious results, respectively. Regarding the mitigation algorithms, even with critical disturbances induced by scintillation, as occurred around 7.3 seconds, there is no sharp increase in Doppler phase RMSEs, showing good mitigation characteristics of the scintillation effects on the LOS dynamics tracking.

\section{ACKNOWLEDGMENT}

This work was partially supported by the Brazilian National Council for Scientific and Technological Development (CNPq) under grant 309248/2018-3 PQ-2, 409865/2018-4, $307255 / 2018-2$ and 205906/2018-4.

\section{REFERENCES}

[1] J. Vilá-Valls, N. Linty, P. Closas, F. Dovis, J. T. Curran, "Survey on Signal Processing for GNSS Under Ionospheric Scintillation: Detection, Monitoring, and Mitigation," Navigation, v. 67, issue 3, pp. 511-536, 2020.

[2] A. O. Moraes, F. S. Rodrigues, W. J. Perrella, and E. R. de Paula, "Analysis of the Characteristics of Low-Latitude GPS Amplitude Scintillation Measured During Solar Maximum Conditions and Implications for Receiver Performance," Surveys in Geophysics, v. 33, no.5, pp. $1107-$ 1131, 2012.

[3] K. S. Jacobsen, and Y. L. Andalsvik, "Overview of the 2015 St. Patrick's day storm and its consequences for RTK and PPP positioning in Norway," J. Space Weather Space Clim., v. 6, no. A9, 2016.

[4] S. Banville, and R. B. Langley, "Mitigating the impact of ionospheric cycle slips inGNSS observations," Journal of Geodesy, v. 87, no. 2, pp. 179-193, 2013.

[5] F. Legrand, C. Macabiau, J.-L. Issler, L. Lestarquit, and C. Mehlen, "Improvement of pseudorange measurements accuracy by using fast adaptive bandwidth lock loops," ION GPS 2000, pp. 2346 - 2356, Salt Lake City, United States, 2000.

[6] R. Xu, Z. Liu, and W. Chen, "Improved FLL-assisted PLL with in-phase pre-filtering to mitigate amplitude scintillation effects," GPS Solutions, v. 19 , pp. 263-276, 2015.

[7] J. Lee, J. Morton, H. Moon and J. Seo, "Monitoring and mitigation of ionospheric anomalies for GNSS-based safety critical systems: A review of up-to-date signal processing techniques," IEEE Signal Processing Magazine, v. 34, no. 5, pp. 96-110, 2017.

[8] J. Van Dierendonck, A.J .and Klobuchar and Q. Hua, "Ionospheric scintillation monitoring using commercial single frequency c/a code receivers," in Proceedings of ION GPS, vol. 93, 1993, pp. 1333-1342.

[9] D. Xu, Y. Morton, D. Akos and T. Walter, "GPS multifrequency carrier phase characterization during strong equatorial ionospheric scintillation," Proceedings of the International Technical Meeting of the Satellite Division of The Institute of Navigation (ION GNSS+), Tampa, FL, 2015.

[10] C. Mayer, B. Belabbas, N. Jakowski, M. Meurer and W. Dunkel, "Ionosphere Threat Space Model Assessment for GBAS," ION GNSS, Savannah, GA, USA, pp. 22-25, September 2009.

[11] J. I. Statman and W. J. Hurd, "An Estimator-Predictor Approach to PLL Loop Filter Design,” IEEE Transactions on Communications, v. 38, no. 10, 1990, pp. 1667-1669.

[12] T. E. Humphreys, M. L. Psiaki and P. M. Kintner, P. M. (2005). "GPS carrier tracking loop performance in the presence of ionospheric scintillations," Proceedings of the International Technical Meeting of the Satellite Division of The Institute of Navigation (ION GNSS), Long Beach, CA, pp. 156-167, 2005.

[13] J. Vilá-Valls, C. Fernandez-Prades, J. López-Salcedo, and G. SecoGranados, "Adaptive GNSS Carrier Tracking Under Ionospheric Scintillation: Estimation vs. Mitigation," IEEE Communications Letters, v. 19, no. 6, pp. 961-964, 2015. 
[14] J. Vilá-Valls, P. Closas, C. Fernandez-Prades and J.T. Curran, "On the Ionospheric Scintillation Mitigation in Advanced GNSS Receivers," IEEE Transactions on Aerospace and Electronic Systems, v. 54, no. 4, pp. 1692-1708, 2018.

[15] J. Vilá-Valls, C. Fernandez-Prades, J. Arribas, J.T. Curran and P. Closas, "On-line Model Learning for Adaptive GNSS Ionospheric Scintillation Estimation and Mitigation," IEEE/ION Position, Location and Navigation Symposium (PLANS), Monterey, CA, 2018, pp. 1167-1172, 2018.

[16] F. Fohlmeister, F. Antreich and J. A. Nossek, "Dual Kalman filtering based GNSS phase tracking for scintillation mitigation," IEEE/ION Position, Location and Navigation Symposium (PLANS), Monterey, CA, pp. 1151-1158, 2018.

[17] F. Fohlmeister, F. Antreich, V. Wilken, M. Kriegel, J. C. M. Mota, A. L. F. de Almeida, F. G. M. Pinheiro, and J. A. Nossek, "Evaluation of Low Latitude Scintillation Data with a Dual Kalman Smoother,' in Proceedings of ITM GNSS 2019, Raston, VA, U.S.A., January 2019.

[18] F. Niu, Y. Morton, S. Taylor, W. Pelgrum and A. J. Van Dierendonck, "Performances of GPS signal observables detrending methods for ionosphere scintillation studies," ION GNSS, Nashville, TN, USA, pp. 34353443, September 2012.

[19] Bar-Shalom, Y., Li, X. R., Kirubarajan, T., Estimation with Applications to Tracking and Navigation, John Wiley and Sons, USA, 2001.

[20] T.E. Humphreys, M.L. Psiaki, P.M. Kintner, "Modeling the effects of ionospheric scintillation on GPS carrier phase tracking", IEEE Trans. Aerosp. Electron. Syst., v. 46(4), pp. 1624-1637, 2010.

[21] Z. Luo, J. Ding, L. Zhao and M. Wu, "An Enhanced Non-Coherent PreFilter Design for Tracking Error Estimation in GNSS Receivers," Sensors (Basel), v. 17, no. 11, pp. 1-23, 2017.

[22] Teunissen, P. J. G. and Montenbruck, O., Springer Handbook of Global Navigation Satellite Systems, Springer International, Switzerland, 2017.

[23] Kaplan, E. D. and Hegarty, C. J., Understanding GPS/GNSS: Principles and Applications, third edition, Artech House, Boston/London, 2017

[24] Borre, K., D. M. Akos, N. Bertelsen, P. Rinder, and S. H. Jensen, A software-defined GPS and Galileo receiver: a single-frequency approach, Springer Science \& Business Media, Birkhaeuser, Boston, 2007.

[25] R. G. Brown and P. Y. C. Hwang, Introduction to Random Signals and Applied Kalman Filtering, third edition, John Wiley \& Sons, New York, US, 1996.

[26] R. E. Kalman and R. S. Bucy, "New Results in Linear Filtering and Prediction Theory," Trans. ASME, Journal of Basic Engineering, v. 83, pp. 95-108, 1961

[27] L. Zhang and Y. T. Morton, "Tracking GPS Signals under Ionospheric Scintillation Conditions," ION GNSS, Savannah, GA, USA, pp. 227-234, September 2009.

[28] M. Susi, D. Borio, "Kalman Filtering with Noncoherent Integrations for Galileo E6-B Tracking," Navigation, v. 67, issue 3, pp. 601-618, 2020

[29] J. A. Del Peral-Rosado; J. A. López-Salcedo; G. Seco-Granados; J. M. López-Almansa; J. Cosmen, "Kalman Filter-based Architecture for Robust and High-sensitivity Tracking in GNSS Receivers," 2010 5th ESAWorkshop on Satellite Navigation Technologies and European Workshop on GNSS Signals and Signal Processing (NAVITEC), pp. 1-8, Noordwijk, The Netherlands, 2010.

[30] T. Soderstrom and P. Stoica: System Identification, Prentice-Hall International, Hemel Hempstead, UK, 1989.

[31] P. C. Young, Recursive Estimation and Time-Series Analysis, second edition, Springer-Verlag, Germany, 2011. 